\title{
The Contributory Impact of Structural Components of Computerized Accounting Systems on the Systems' Overall Performance in Small and Medium Enterprises
}

\author{
Akamanwam Effiong Itang, $\mathrm{PhD}$ \\ Consulting Accounting, Florina Limited (Seconded to ENI Nigeria), POBox 923, Port Harcourt, Nigeria
}

\begin{abstract}
In today's digital era, the application of computers and related technologies in the accounting function amongst business organizations has increased, giving rise to substantial scholarly and practical debates on the subject of computerized accounting systems. Several studies have, therefore, been conducted on computerized accounting systems or accounting information systems in recent times. However, existing studies are focused on the adoption, usage, and benefits of the systems. No extant studies has attempted to explore the structural components or subsystems of computerized accounting systems and how these components contribute to the overall performance of the systems. To fill this gap, the present study has attempted to explore the contributory impact of the structural components (subsystems) of computerized accounting systems to the systems' overall performance. This study, therefore, decomposed computerized accounting systems into five components and, employing structural equation model (SEM), sought to determine the contributory impact of each of the components to the holistic performance and effectiveness of the systems, with a focus on small and medium enterprises in Nigeria. The quasi-experimental, cross-sectional survey was employed in the study and questionnaires were administered to 370 firms that were selected using the stratified random sampling technique. The results of the study indicates that all five components, individually, made significant contribution to the overall performance of computerized accounting systems (CAS). The study, therefore, concluded that each of the structural components of CAS, namely internal controls, automated data-processing, relational database, automated reporting, and enhancing technologies, individually, contributed significantly to the overall performance of the systems. Hence, all the five components are necessary in the design, modelling, choice, and adoption of computerized accounting systems for SMEs. The study is significant as it contributes empirically to the body of knowledge in the subject. Options for further research are also suggested for the validation and, or, extension of the study's results.
\end{abstract}

Keywords: Accounting information systems, Automated data-processing, Automated reporting, Computerized accounting systems, Enhancing technologies, Internal controls, Relational database, Small and medium enterprises, Structural characteristics model, Structural components, Subsystems, Systems performance

DOI: $10.7176 / \mathrm{RJFA} / 12-18-11$

Publication date:September $30^{\text {th }} 2021$

\section{Introduction}

In today's digital era, the application of computers and related technologies in the accounting function amongst business organizations has increased, giving rise to substantial scholarly and practical debates on the subject of computerized accounting systems. A system is "a set of elements standing in interrelation among themselves and with the environment" (von Bertalanffy, 1972, p.417), with the aim of achieving a sole objective. In other word, every system "comprises of components (or subsystems) that are interrelated, and which perform their functions towards the achievement of the objectives of the entire system" (Itang, 2020, p. 40). Therefore, computerized accounting systems are designed with various interrelated structural components or subsystems that work together towards an effective and efficient execution of the accounting function, from collecting, recording, organizing, summarizing, and analyzing of business transactions, to the reporting of financial information to stakeholders. Based on the general systems theory, Itang (2020) developed the structural characteristics model (SCM) for computerized accounting systems, which identified five major structural components or subsystems, namely internal controls component, automated data-processing component, relational database component, automated reporting component, and enhancing technologies component. The SCM is significant as it considers the functional components of the system instead of the perceived benefits of the systems.

In line with the general systems theory, each of the subsystems or components of computerized accounting system indicated in the Itang's (2020) structural characteristics model are expected to function in synergy with each other to ensure the holistic performance of the entire system. This implies that the optimal performance of the computerized accounting system would depend, to a great extent, on the contributory performance and synergy of its various components. While several studies have been conducted on computerized accounting systems or accounting information systems in recent times, the existing studies are focused on the adoption, usage, and benefits of the systems No extant study has been identified by the researcher attempting to explore the 
structural components or subsystems of computerized accounting systems and how these components contribute to the overall performance of the systems. Hence, there is a gap in the literature and body of knowledge in the field of accounting information systems regarding the contribution of system components to the general performance of computerized accounting systems. This study, therefore, aims to fill this gap by exploring the extent to which each of the structural components of computerized accounting systems, as identified by Itang (2020), contributes to the overall performance of the system.

\subsection{Research question}

To guide the study the following research questions and hypotheses were considered.

To what extent does each of the structural components of computerized accounting systems contribute to the overall performance of the system?

\subsection{Research hypotheses}

To adequately address the research question indicated above, the following five hypotheses, stated in their null forms, were tested.

$\mathrm{H}_{0} 1$ : Internal controls component does not significantly contribute to the overall performance of computerized accounting systems.

$\mathrm{H}_{\mathrm{o}}$ 2: Automated data-processing component does not significantly contribute to the overall performance of computerized accounting systems.

$\mathrm{H}_{\mathrm{o}} 3$ : Relational database component does not significantly contribute to the overall performance of computerized accounting systems.

$\mathrm{H}_{0} 4$ : Automated reporting component does not significantly contribute to the overall performance of computerized accounting systems.

$\mathrm{H}_{0}$ 5: Enhancing technologies component does not significantly contribute to the overall performance of computerized accounting systems.

This study is significant as it provides empirical evidence on the contribution of each of the structural components or subsystems of computerized accounting systems to the holistic performance of the systems. The study, therefore, contributes to the literature and body of knowledge in accounting information systems in this regard.

\section{Literature Review}

\subsection{Theoretical Framework}

As indicated by Adom et al. (2018), a theoretical framework provides a blueprint based on existing theory (or theories), which guides the entire research process. This study is, therefore, framed by the general systems theory, which views an entity as a whole, with a focus on the interaction and relationship between the various components of the entity towards its overall outcome (von Bertalanffy, 1968, 1972). The application of systems theory in business research is quite significant as it would enhance effective research and application of outcomes since it considers the study phenomenon holistically as well as the reductionist view of individual elements of the entity (Mele et al., 2010). Underpinned by the general systems theory, the study explores how the various structural components of computerized accounting systems contributes individually to the overall performance of the systems.

\subsection{Overview of Computerized Accounting Systems}

A computerized accounting system, also known as electronic accounting system or accounting software, "consists of the application of computers and other technological innovation tools in the accounting process" (Itang, 2021, p.29). Marivic (2009) noted that computerized accounting systems are methods and schemes employed in recording, organizing, summarizing, and analyzing business transactions, as well as interpreting and communicating financial information to various stakeholders with the use of computers and related technologies. Computerized accounting systems are designed and developed in various formats, including standalone software, network-based packages, web/cloud-based applications, and as parts of ERP solutions (Gupta et al., 2017; Itang, 2020, 2021). In line with the purpose of accounting as an information systems (American Institute of Certified Public Accountants [AICPA], 1996), computerized accounting systems are designed to enhance the production and communication of useful financial information to various stakeholders for sound decision making. Therefore, the various components of the computerized accounting system are expected to work in synergy towards achieving this purpose.

\subsection{Structural Components of Computerized Accounting Systems}

Based on the general systems theory, Itang (2020) maintained that a computerized accounting system is a consolidation of several components or subsystems that form the system's architectural framework. Paganini 
(2019) indicated that the system's architecture defines the logical organization of software into subsystems to enable the system to perform optimally and reliably. Computerized accounting systems, therefore, consist of integrated components that take care of the various stages and processes in the accounting cycle, ranging from financial data collection and input, through processing, to storage and report of financial information. Assefa et al. (2020), while proposing a possible model of accounting information systems for small and medium enterprises, identified three subsystems, namely transaction processing, internal control, and reporting. However, Itang (2020) presents a more robust model that identified five structural components or subsystems of computerized accounting systems, namely internal controls, automated data-processing, relational database, automated reporting, and enhancing technologies components, each of which is conceptualized in the following subsections.

Internal Controls Component. Internal control is defined as "a process effected by an entity's board of directors, management, and other personnel, designed to provide, reasonable assurance regarding the achievement of objectives relating to operations, reporting, and compliance" (The Committee of Sponsoring Organizations of the Treadway Commission [COSO], 2013, P.3). Based on the COSO (2013) definition of internal control, the function of internal controls component of computerized accounting systems is to ensure that the performance of the system enhances effective and efficient operation of the organization in general and the accounting function in particular, good quality financial reporting, and adequate compliance with established policies, laws, rules, and regulation. Hence, the internal controls component is a very crucial structural component, particularly in terms of its role in effective financial reporting.

Automated Data-processing Component. The automated data-processing component or subsystem is responsible for the systems' ability to perform accounting tasks and processes automatically, without human intervention (Itang, 2020). This component enables the computerized accounting system to validate, and process financial data, post transactions, as well as balance and reconcile accounts seamlessly (Anggraeni, 2016; Intuit Inc., 2018).

Relational Database Component. The relational database component is the subsystem responsible for the storage, maintenance, and utilization of data and information in the system (Ramakrishnan \& Gehrke, 2003). Therefore, every computerized accounting system is characterized by a relational database management subsystem (RDBMS) that maintains the relationship between accounting records and files within the system, as well as ensures the independence, integrity, security, scalability, and concurrence of data (Agung, 2015; Itang, 2020).

Automated Reporting Component. This component is responsible for the ability of the computerized accounting system to automatically generate reports in various formats and for various purposes (Intuit Inc., 2018). For the reporting subsystem to perform optimally, the relational database management subsystem must also work effectively as it acts as the custodian of the data and information necessary for the generation of every form of report.

Enhancing Technologies Component. The enhancing technologies component comprised of all the relevant technological tools and devices that are not part of the accounting software architecture, but are required by the system to function effectively and efficiently (Itang, 2020). These technological tools include various forms of computer devices and accessories, such as hardware, firmware, applications, network infrastructure, printers, scanners, emailing applications, etc. (Anggraeni, 2016; Ghasemi, et al., 2011; Gupta et al., 2017).

\subsection{Conceptual Framework}

Considering the foregoing review of literature on the structural components of computerized accounting systems, this study is conceptually framed by Itang's (2020) structural characteristic model (SCM). Based on the SCM, the following conceptual model was developed for the study, where the components of computerized accounting systems (CAS), namely internal controls, automated data-processing, relational database, automated reporting, and enhancing technologies, are represented by IC, DP, DB, RO, and ET, respectively. 


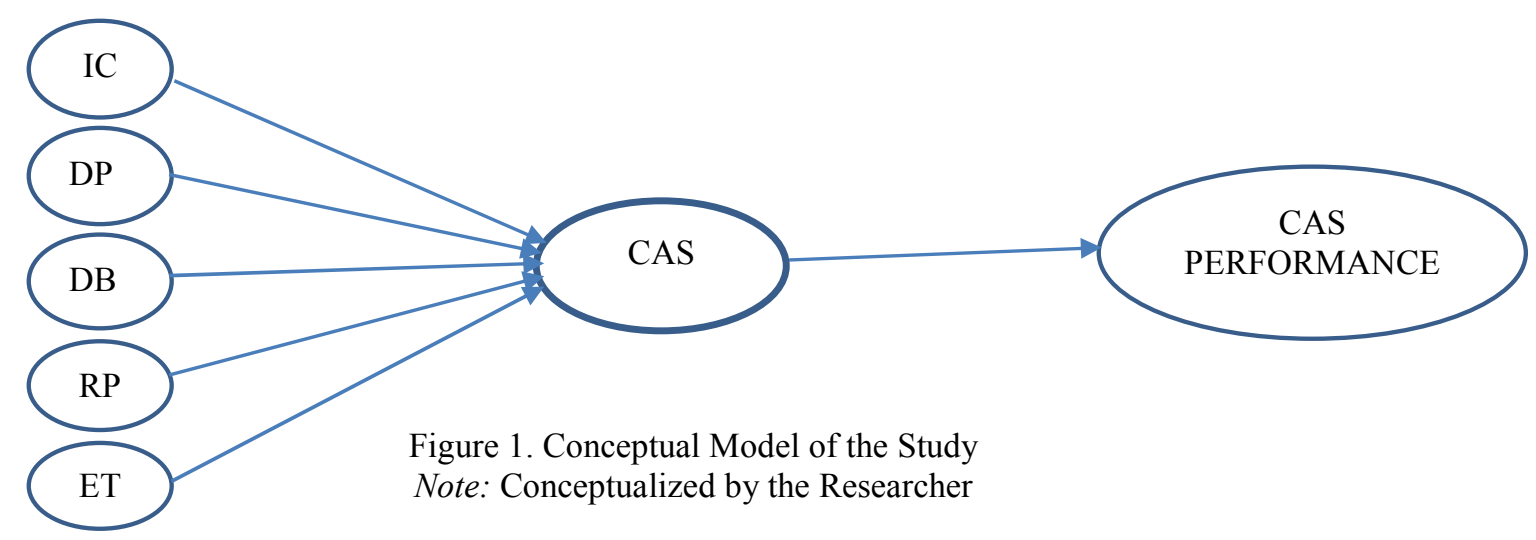

\section{Methods}

\subsection{Study Design}

The study is quasi-experimental, and was conducted using the cross-sectional survey design. The survey research design is advantageous as it aids effective collection of opinions and explanations about the phenomenon under investigation, as well as provides generalizable study results ( Selhin et al., 2016; Kpolovie, 2016).

\subsection{Study population and Sample}

The population of the study was small and medium enterprises (SMEs) in Nigeria. However, the sampling frame was restricted to SMEs within the south-south zone of Nigeria. As indicated by the Small and Medium Enterprises Development Agency of Nigeria (SMEDAN), there are 9,276 SMEs in the south-south zone (SMEDAN, 2013). Using the stratified random sampling technique, a total of 370 firms were selected for the study, based on the Krejcie and Morgan's sample size determination table (Krejcie \& Morgan, 1970, p.608).

\subsection{Operationalization of Study Variables}

The components of computerized accounting systems were operationalized based on Itang's (2020) structural characteristics model. Hence, the various components of computerized accounting systems (CAS) - internal controls, automated data-processing, relational database, automated reporting, and enhancing technologies were measured using five indicators each, while CAS performance was measured with a single indicator. A total of 26 indicators $(25$ for CAS components and one for CAS performance. Table 1 highlights the various CAS components and their measurement indicators, as well as the indicator of CAS performance.

\subsection{Data Collection and Analysis}

The research data was collected using survey questionnaires administered to one finance/accounting personnel in each of the 370 sampled SMEs. A total of 223 useful responses where received and used in the analysis. The researcher employed the structural equation model (SEM) technique in analyzing the collected data. SEM has the advantage of modelling relationships between multiple variables simultaneously as well as enabling the testing of several hypotheses in a single systematic analysis (Bagozzi \& Yi, 2012). More so, the study employs both unobserved (latent) and observed (indicator) variables, and SEM is appropriate as it allows the use of indicators to measure the latent variables as well as enhances the estimation of the statistical significance of constructs and the relationship between variables (Lorraine et al., 2011; Al-Dweikat \& Nour, 2018).

\subsection{Validity and Reliability Tests}

The study adopted the CAS measurement tool, the structural characteristics model, developed by Itang (2020), which has been indicated to have strong validity and high reliability for the assessment of CAS quality. 
Table 1. Operationalization of CAS Components

\begin{tabular}{|c|c|}
\hline CAS Component & Indicators \\
\hline \multirow[t]{5}{*}{ Internal controls (IC) } & Access control \\
\hline & Segregation of duties \\
\hline & Accuracy checks \\
\hline & Security controls \\
\hline & Audit trail \\
\hline \multirow[t]{5}{*}{ Automated data-processing (DP) } & Seamless processing \\
\hline & Data validation \\
\hline & Transaction posting \\
\hline & Accounts balancing \\
\hline & Accounts reconciliation \\
\hline \multirow[t]{5}{*}{ Relational database (DB) } & Large storage \\
\hline & Data maintenance \\
\hline & Data independence \\
\hline & Backup and recovery \\
\hline & Concurrent access \\
\hline \multirow[t]{5}{*}{ Automated reporting (RP) } & Seamless reporting \\
\hline & Trial balance and GL reports \\
\hline & Financial statements \\
\hline & Multiple format options \\
\hline & Comparative reporting \\
\hline \multirow[t]{5}{*}{ Enhancing technologies (ET) } & Network configuration \\
\hline & Cloud computing \\
\hline & Document upload \\
\hline & POS interface \\
\hline & Email/SMS interface \\
\hline CAS performance (SP) & Information quality \\
\hline
\end{tabular}

Note: Adopted by the Researcher from Itang (2020)

\section{Study Results}

\subsection{Evaluation of the Reflective Measurement Model for CAS Components}

Evaluation of the reflective measurement model for CAS components was based on the factor loadings for each of the components' indicators as well as the composite reliability (CR) and average variance explained (AVE) for respective components. Table 2 shows the reflective measurement model evaluation for CAS components, which highlights that all the item loadings exceeded 0.50 and were significant at $\mathrm{P}<0.000$. The reliability of the measurement scales were validated using the CR and the AVE, the duo of which have been indicated to help ascertain the level of association that exist between study constructs and their respective indicators. The relatively high values of CR and AVE indicated by the results shown in Table 2 indicated a strong and satisfactory reliability and convergent validity.

Table 2. CAS Reflective Measurement Model Assessment

\begin{tabular}{lllllll}
\hline & $\mathrm{M}$ & $\mathrm{SD}$ & Loadings & CR & AVE & $\sqrt{\text { AVE }}$ \\
\hline Internal Controls & - & - & - & 0.887 & 0.613 & 0.783 \\
Access controls & 4.73 & 0.794 & 0.810 & & & \\
Segregation of duties & 4.61 & 0.862 & 0.814 & & & \\
Accuracy checks & 4.34 & 0.977 & 0.655 & & & \\
Security controls & 4.54 & 0.899 & 0.851 & & & \\
Audit trail & 4.60 & 0.752 & 0.771 & & & \\
\hline Automated data-processing & - & - & - & 0.922 & 0.703 & \\
Seamless processing & 4.60 & 0.804 & 0.795 & & & \\
Data validation & 4.45 & 0.797 & 0.890 & & & \\
Transaction Posting & 4.28 & 1.198 & 0.857 & & & \\
Accounts balancing & 4.52 & 0.895 & 0.859 & & & \\
Accounts reconciliation & 4.20 & 0.935 & 0.787 & & & \\
\hline
\end{tabular}




\begin{tabular}{|c|c|c|c|c|c|c|}
\hline & $\mathrm{M}$ & SD & Loadings & CR & AVE & $\sqrt{A V E}$ \\
\hline Relational Database & - & - & - & 0.913 & 0.681 & 0.825 \\
\hline Large storage & 4.80 & 0.402 & 0.769 & & & \\
\hline Data maintenance & 4.74 & 0.442 & 0.688 & & & \\
\hline Data independence & 4.50 & 0.734 & 0.933 & & & \\
\hline Backup and recovery & 4.52 & 0.709 & 0.925 & & & \\
\hline Concurrent access & 4.58 & 0.717 & 0.785 & & & \\
\hline Automated reporting & - & - & - & 0.882 & 0.600 & 0.775 \\
\hline Seamless reporting & 4.74 & 0.567 & 0.766 & & & \\
\hline Trial balance/GL reports & 4.82 & 0.453 & 0.750 & & & \\
\hline Financial statements & 4.26 & 0.950 & 0.796 & & & \\
\hline Multiple formats options & 4.46 & 0.769 & 0.820 & & & \\
\hline Comparative reporting & 4.39 & 0.887 & 0.738 & & & \\
\hline Enhancing technologies & - & - & - & 0.915 & 0.684 & 0.827 \\
\hline Network configuration & 4.57 & 0.743 & 0.741 & & & \\
\hline Cloud computing & 4.28 & 0.942 & 0.794 & & & \\
\hline POS interface & 3.99 & 1.187 & 0.877 & & & \\
\hline Documents upload & 4.05 & 0.904 & 0.829 & & & \\
\hline Email/SMS interface & 4.33 & 0.992 & 0.885 & & & \\
\hline
\end{tabular}

Note: Extracted by the Researcher from the research data analysis output

\subsection{Structural Model and Results}

The structural model results represented by the structural path parameter estimates is shown in Figure 2 . The parameter estimates are represented by the standardized beta $(\beta)$ values, which range from $0-1$. The beta $(\beta)$ values may be positive or negative $(-1 \leq \beta \leq 1)$ and the closer the value is to \pm 1 , the stronger the relationship; while \pm 1 indicates a perfect relationship. The variance explanatory power (VE) indicates the extent to which an independent (exogenous) variable can predict the dependent (endogenous) variable. Table 3 is a tabular presentation of the structural model results with their respective variance inflation factor (VIF). The VIF is a collinearity test parameter and as shown in Table 3, the VIF values ranging from 1.905 to 3.998 , indicating a minimal collinearity among the indexes. The acceptable VIF threshold is $0.5-10.0$.

From Figure 2 and Table 3, the beta $(\beta)$ values explain the level of contribution of each of the structural components of CAS to its overall performance. Therefore, internal controls component contributes $23.8 \%$ to the performance of the entire system, while automated data-processing contributes $27.8 \%$. Similarly, the relational database, automated reporting, and enhancing technologies components contribute $16.9 \%, 20.3 \%$, and $30.4 \%$, respectively, to the overall CAS performance. This results indicate that the enhancing technologies component contributes the most to the performance of CAS, while the relational database component contributes the least.

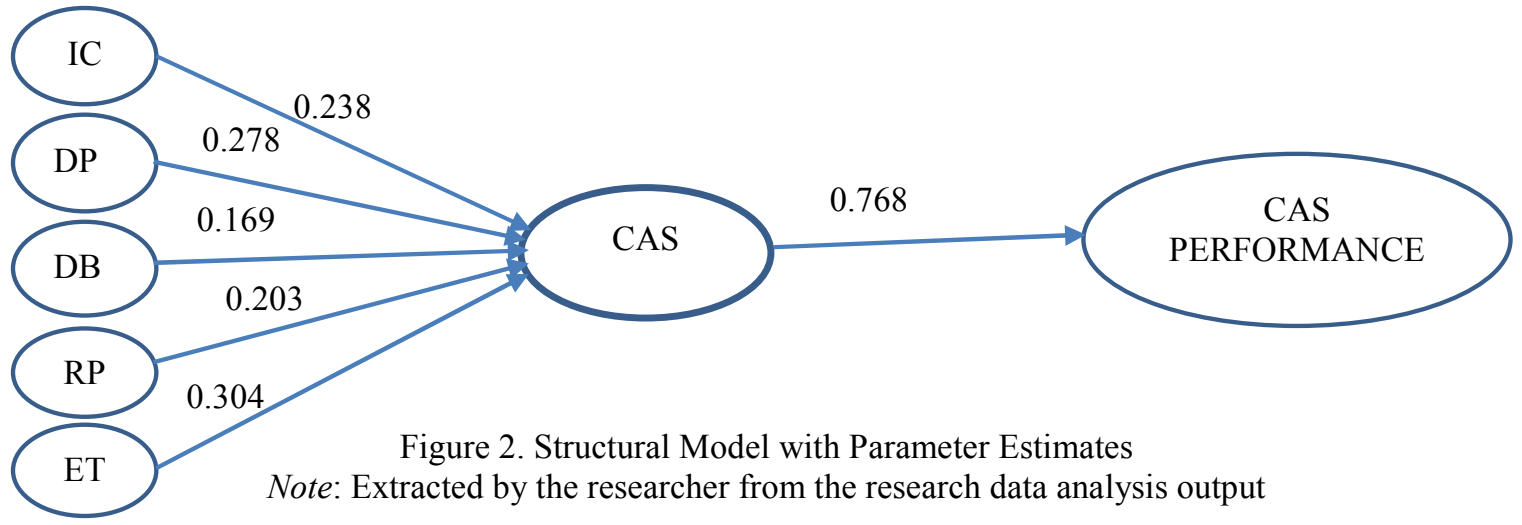


Table 3. Tabular Presentation of the Structural Model Results

\begin{tabular}{llll}
\hline CAS Component & $\beta$ & VIF & $\mathrm{P}$ \\
\hline Internal controls & 0.238 & 1.905 & $\mathrm{P}<0.05$ \\
Automated data-processing & 0.278 & 2.672 & $\mathrm{P}<0.05$ \\
Relational database & 0.169 & 3.998 & $\mathrm{P}<0.05$ \\
Automated reporting & 0.203 & 2.920 & $\mathrm{P}<0.05$ \\
Enhancing technologies & 0.304 & 3.832 & $\mathrm{P}<0.05$ \\
Note: Extracted by the researcher from the research data analysis output
\end{tabular}

\subsection{Hypotheses Test Results}

Based on the parameter estimates obtained from the structural model, the results of the five hypothesized relationships are explained as follows.

Hypothesis 1 Result. The first hypothesis was that internal controls component does not significantly contribute to the overall performance of computerized accounting systems. This relationship is estimated with a standardized beta of 0.238 , which indicates that internal controls component contributes about $24 \%$ to the overall performance of CAS. Since the fair contribution of each of the five components to the overall performance of CAS would have been $20 \%$, the contribution of about $24 \%$ by internal controls component is quite significant. Hence, the result shows that internal controls component significantly contribute to the overall performance of CAS. The null hypothesis that internal controls component does not significantly contribute to the overall performance of computerized accounting systems is, therefore, rejected.

Hypothesis 2 Result. The second hypothesis was that automated data-processing component does not significantly contribute to the overall performance of computerized accounting systems. This relationship is estimated with a standardized beta of 0.278 , which indicates that automated data-processing component contributes about $28 \%$ to the overall performance of CAS. Since the fair contribution of each of the five components to the overall performance of CAS would have been $20 \%$, the contribution of about $28 \%$ by automated data-processing component is quite significant. Hence, the result shows that automated dataprocessing component significantly contribute to the overall performance of CAS. The null hypothesis that automated data-processing component does not significantly contribute to the overall performance of computerized accounting systems is, therefore, rejected.

Hypothesis 3 Result. The third hypothesis was that relational database component does not significantly contribute to the overall performance of computerized accounting systems. This relationship is estimated with a standardized beta of 0.169 , which indicates that relational database component contributes about $17 \%$ to the overall performance of CAS. Since the fair contribution of each of the five components to the overall performance of CAS would have been $20 \%$, the contribution of about $17 \%$ by relational database component is quite close to $20 \%$ and is, therefore, significant. Hence, the result shows that relational database component significantly contribute to the overall performance of CAS. The null hypothesis that relational database component does not significantly contribute to the overall performance of computerized accounting systems is, therefore, rejected.

Hypothesis 4 Result. The fourth hypothesis was that automated reporting component does not significantly contribute to the overall performance of computerized accounting systems. This relationship is estimated with a standardized beta of 0.203 , which indicates that automated reporting component contributes about $20 \%$ to the overall performance of CAS. Since the fair contribution of each of the five components to the overall performance of CAS would have been $20 \%$, the contribution of about $20 \%$ by automated reporting component is significant. Hence, the result shows that automated reporting component significantly contribute to the overall performance of CAS. The null hypothesis that automated reporting component does not significantly contribute to the overall performance of computerized accounting systems is, therefore, rejected.

Hypothesis 5 Result. The fifth hypothesis was that enhancing technologies component does not significantly contribute to the overall performance of computerized accounting systems. This relationship is estimated with a standardized beta of 0.304 , which indicates that enhancing technologies component contributes about $30 \%$ to the overall performance of CAS. Since the fair contribution of each of the five components to the overall performance of CAS would have been $20 \%$, the contribution of about $30 \%$ by enhancing technologies component is very significant. Hence, the result shows that enhancing technologies component significantly contribute to the overall performance of CAS. The null hypothesis that enhancing technologies component does not significantly contribute to the overall performance of computerized accounting systems is, therefore, rejected. 
Table 4. Summary of Hypotheses Test Results

\begin{tabular}{llllll}
\hline Hypothesis & Structural Path & P-Value & $\beta$ & VE & Decision \\
\hline $\mathrm{H}_{0} 1$ & IC-CAS & 0.000 & 0.238 & 0.057 & Rejected \\
$\mathrm{H}_{0} 2$ & DP-CAS & 0.000 & 0.278 & 0.077 & Rejected \\
$\mathrm{H}_{0} 3$ & DB-CAS & 0.000 & 0.169 & 0.029 & Rejected \\
$\mathrm{H}_{0} 4$ & RP-CAS & 0.000 & 0.203 & 0.041 & Rejected \\
$\mathrm{H}_{0} 5$ & ET-CAS & 0.000 & 0.304 & 0.092 & Rejected \\
\hline
\end{tabular}

Note: Extracted by the researcher from research data analysis output

\section{Discussion and Conclusion}

\subsection{Discussion of Results}

Computerized accounting systems (CAS) are characterized by components or subsystems. This study explored the contributory impact of each of the components of CAS by employing the structural characteristics model (Itang, 2020), which identifies five subsystems of CAS, namely internal controls, automated data-processing, relational database, automated reporting, and enhancing technologies components. The results of the study indicate that each of the five components of CAS contributed significantly to the overall performance of the systems. Specifically, the internal controls component contributed about $24 \%$ to the holistic performance of the computerized accounting systems, while automated data-processing, relational database, automated reporting, and enhancing technologies components contributed about $28 \%, 17 \%, 20 \%$, and $30 \%$, respectively.

Therefore, enhancing technologies is indicated to have the most contribution $(30 \%)$ to the performance of the computerized accounting systems. This finding could be attributed to the enhancing technologies component provides the basic enabling infrastructure for data transformation, transmission, and communication within the computerized accounting systems environments. The automated data-processing subsystem is the second most contributing component, contributing about $28 \%$, to the overall performance of CAS. This is not surprising as the automated data-processing component is the domain for the actual computation, simulation, verification, transformation, and analysis of financial data within the accounting system. The other components, namely internal controls, relational database, and automated reporting contributed about 24\%, 17\%, and $20 \%$, respectively, to the overall performance of the computerized accounting systems. Hence, the relational database component had the least contribution to the performance of the holistic performance of computerized accounting systems. The low contributory impact of relational database could be attributed to the fact that the subsystem exists as a back-end facility that is relatively not influenced by environmental factors, with responsibility for storage, maintenance, and retrieval of financial data and information (Ramakrishnan \& Gehrke, 2003).

One would have expected the internal controls component of CAS to be the most contributing component, considering the significance of internal controls over financial reporting as advocated by the Section 404 of the Sarbanes-Oxley Act (United States, 2002) and COSO internal controls integrated framework (COSO, 2013). However, all the components or subsystems of CAS are indicated to have significant contribution to the overall performance of the systems, which shows that all the five components are important in the design of computerized accounting systems and indispensable in the effectiveness and efficiency of the systems. This result supports the position of Assefa et al. (2020) that the internal control, processing, and reporting subsystems are essential in designing a model of accounting information systems for SMEs.

\subsection{Conclusion and Recommendations}

The study aimed to explore the contributory impact of the various components of computerized accounting systems to the overall performance of the systems. The five components employed in the study were based on the structural characteristics model, namely internal controls, automated data-processing, relational database, automated reporting, and enhancing technologies (Itang, 2020). Five hypotheses, each proposing that individual component of CAS does not contribute significantly to the overall performance of the systems, were tested in the study. The results of the study indicate that all five components, individually, contribute significantly to the overall performance of computerized accounting systems. All five null hypotheses were, therefore, rejected and the alternate forms accepted. The study, therefore, concluded that each of the structural components of CAS, namely internal controls, automated data-processing, relational database, automated reporting, and enhancing technologies, individually, contributed significantly to the overall performance and effectiveness of the systems. Hence, all the five components are adjudged necessary in the design, modelling, choice, and adoption of computerized and accounting systems for SMEs.

This study provides empirical evidence on the contribution of each of the structural components or subsystems of computerized accounting systems to the overall performance of the systems and, therefore, contributes to the literature and body of knowledge in accounting information systems in this regard. It is recommended that management and accounting officers in SMEs should pay attention to the enhancing 
technologies components of the computerized accounting systems and ensure that relevant technological tools, devices, applications, and infrastructure necessary for the optimal performance of the accounting systems are provided to the systems. This is very necessary as the enhancing technologies component has been indicated to have the most contributory impact on the overall effectiveness of computerized accounting systems. They should also ensure that their computerized accounting systems have strong internal controls, reliable data-processing and relational database, and good reporting subsystems.

The study is, however, limited by the fact that it focused on small and medium enterprises. Nevertheless, the results of the study could be generalized to other economic sectors or larger organizations. It is suggested that similar studies could be carried out using different sampling frames or larger firms to validate and, or, extend the outcome of this study.

\section{References}

Adom, D., Hussein, E. K., \& Agyem, J. A. (2018). Theoretical and conceptual framework: Mandatory ingredients of a quality research. International Journal of Scientific Research, 7(1), 438-441.

Agung, M. (2015). Accounting information systems and improvement on financial reporting. International Journal of Recent Advances in Multidisciplinary Research, 2(11), 950-957.

Al-Dweikat, M. F. S. \& Nour, M. I. (2018). A structural equation model for analyzing the impact of strategic management accounting techniques on quality of financial information. International Journal of Economics and Finance, 10(4), 62-71.

American Institute of Certified Public Accountants (1996). AICPA professional standards: Code of professional conduct and bylaws as of June 1, 1996. AICPA Professional Standards - 116. Retrieved from https://egrove.olemiss.edu/aicpa_prof/116

Anggraeni, A. F. (2016). Correlation between information technology and management information systems quality. International Journal of Scientific \& Technology Research, 5(6), 168-172.

Assefa, M., Rao, Y. J., \& Yohannes, A. (2020). Designing of accounting information systems for small and medium enterprises: Application of PLS_SEM. International Journal of Sciences: Basic and Applied Research, 54(3), 124-139.

Bagozzi, R. P., \& Yi, Y. (2012). Specification, evaluation, and interpretation of structural equation models. Journal of the Academy of Marketing Science, 40, 8-34. https://doi.org/10.1007/s11747-011-0278-x

Committee of Sponsoring Organizations of the Treadway Commission. (2013). Internal control - integrated framework: Executive summary. Retrieved 19 May 2016 from http://www.coso.org/documents/990025P_Executive_Summary_final_may20_e.pdf

Ghasemi, M., Shafeiepour, V., Aslani, M., \& Barvayeh, E. (2011). The impact of information technology (IT) on modern accounting systems. Procedia - Social and Behavioural Sciences, 28, 112-116.

Gupta, D., \& Jain, M. (2017). Impact of cloud accounting on business performance. International Research Journal of Commerce, Arts and Science, 8(12), 321-329.

Intuit Inc. (2018). QuickBooks small business user guide: Starting out with QuickBooks online. Intuit Australia. $\begin{array}{llll}\text { Retrieved } & 16 & \text { October } & 2019\end{array}$ https:/quickbooks.intuit.com/content/dam/intuit/quickbooks/i18n/en/Australia/pdf/QuickBooks-SmallBusiness-User-Guide.pdf

Itang, A. E. (2020). Computerized accounting systems: Measuring structural characteristics. Research Journal of Finance and Accounting, 11(16), 38-54. doi.org/10.7176/RJFA/11-16-05

Itang, A. E. (2021). Computerized accounting systems and financial reporting quality in small and medium enterprises in Nigeria (Unpublished doctoral dissertation). LIGS University, Honolulu, Hawaii, United States.

Kpolovie, P. J. (2016). Excellent research methods. Partridge Publishing.

Krejcie, R. V. \& Morgan, D. W. (1970). Determining sample size for research activities. Educational and Psychological Measurement, 30(3), pp. 607-610.

Lorraine, L., Stacie, P., Dutch, F., \& Shani, R. (2011). On the use of partial least squares path modelling in accounting research. International Journal of Accounting Information Systems, 12, 305-328.

Marivic, A. (2009). Evaluating the security of computerized accounting information systems: An empirical study on Egyptian banking industry (Unpublished doctoral thesis). Aberdeen University, UK.

Mele, C., Pels, J., \& Polese, F. (2010). A brief review of systems theories and their management applications. Service Science, 2(1/2), 126-135.

Paganini, C. (2019). Primer: Understanding software and system architecture. Online: The New Stack. Retrieved 13 April 2020 from https://thenewstack.io/primer-understanding-software-and-system-architecture/

Ramakrishnan, R., \& Gehrke, J. (2003). Database management systems (3rd ed). McGraw-Hill.

Salhin, A., kyiu, A., Taheri, B., Porter, C., Valantasis-Kanellos, N., \& Konig, C. (2016). Quantitative data gathering methods and techniques. In A. Paterson, D. Leung, W. Jackson, R. Maclntosh, \& K. O'Gorman 
(Eds.). Research methods for accounting and finance (pp. 168-186). Goodfellow Publishers Limited.

Small and Medium Enterprises Development Agency of Nigeria [SMEDAN]. (2013). SMEDAN and National Bureau of Statistics collaborative survey: Selected findings (2013). SMEDAN. Retrieved 20 May 2018 from https://smedan.gov.ng/images/PDF/2013-MSME-Survey-Summary-Report.pdf

United States. (2002). Sarbanes-Oxley Act of 2002: Conference report (to accompany H.R. 3763). Washington, D.C.: U.S. G.P.O..

von Bertalanffy, L. (1968). General systems theory: Foundations, development, applications. George Braziller Inc.

von Bertalanffy, L. (1972). The history and status of general systems theory. The Academy of Management Journal, 15(4), 407-426. 